05,11

\title{
Кинетика структурных фазовых переходов в сплаве $\mathrm{Fe}_{80.5} \mathrm{Ga}_{19.5}$
}

\author{
(C) О.О. Павлухина ${ }^{1}$, В.В. Соколовский ${ }^{1,2}$, В.Д. Бучельников ${ }^{1,2}$, М.А. Загребин ${ }^{1,2}$ \\ 1 Челябинский государственный университет, \\ Челябинск, Россия \\ ${ }^{2}$ Национальный исследовательский технологический университет „МИСиС“, \\ Москва, Россия \\ E-mail: pavluhinaoo@mail.ru
}

Поступила в Редакцию 8 июля 2021 г.

В окончательной редакции 13 июля 2021 г.

Принята к публикации 16 июля 2021 г.

\begin{abstract}
Данная работа посвящена моделированию методом Монте-Карло кинетики фазовых переходов типа порядок-беспорядок в сплаве $\mathrm{Fe}_{80.5} \mathrm{Ga}_{19.5}$, используя гамильтониан Блюме-Эмери-Гриффитса. Исследование фазовых превращений реализовано в две стадии: (1) разная скорость охлаждения (различное число шагов Монте-Карло при постоянном значении энергии связи); (2) изотермический отжиг (энергия связи зависит от числа шагов Монте-Карло при фиксированной температуре). В первом случае, высокая скорость охлаждения приводит к одному фазовому переходу $\mathrm{A} 2 \rightarrow \mathrm{D}_{3}+\mathrm{A} 2$ при $700^{\circ} \mathrm{C}$, тогда как медленное охлаждение влечет к переходу $\mathrm{A} 2 \rightarrow \mathrm{D}_{3}+\mathrm{A} 2$ при $700^{\circ} \mathrm{C}$ через смесь фаз $\mathrm{A} 2, \mathrm{D}_{3}, \mathrm{~B} 2$ и $\mathrm{L}_{2}$ в интервале температур от 850 до $700^{\circ} \mathrm{C}$. Во втором случае показано увеличение объемной доли $\mathrm{L} 1_{2}$ фазы с увеличением длительности отжига при $750^{\circ} \mathrm{C}$. На основании полученных данных построена термокинетическая диаграмма фазовых превращений.
\end{abstract}

Ключевые слова: метод Монте-Карло, Fe-Ga, фазовые переходы.

DOI: 10.21883/FTT.2021.11.51579.08s

\section{1. Введение}

Сплавы на основе Fe-Ga привлекают внимание ученых по всему миру благодаря ряду интересных с точки зрения практического применения свойств. В частности, данные сплавы используются при изготовлении сенсоров и датчиков давления [1-4]. Отметим, что в сплавах $\mathrm{Fe}-\mathrm{Ga}$ наблюдаются рекордные значения магнитострикции среди сплавов на основе железа. Наиболее интересными свойствами обладают сплавы с содержанием галлия от 19 до 27 ат.\%, так как в данном концентрационном диапазоне сплавы обладают наилучшими функциональными свойствами. Так же отметим, что в данной области наблюдается два пика магнитострикции $[4,5]$. В работе [6] представлены исследования фазовой диаграммы для сплава $\mathrm{Fe}-\mathrm{Ga}$, из которой следуют следующие фазы: A2, B2, D0 $3, \mathrm{D}_{19}, \mathrm{~L}_{2}$ в зависимости от содержания $\mathrm{Ga}$.

В работе [7] исследована структура и фазовый состав сплавов $\mathrm{Fe}_{100-x} \mathrm{Ga}_{x}$ с содержанием $\mathrm{Ga}$ от 15 до 45 at.\% в зависимости от влияния длительного отжига диапазоне температур 300-600 С. Показано, что фаза А2 является преобладающей в интервале концентраций $15<x<21$, фаза $\mathrm{L1}_{2}$ - в интервале концентраций $21<x<33$, тогда как при большем содержании $\mathrm{Ga}$ появляется смесь фаз $\mathrm{Fe}_{6} \mathrm{Ga}_{5}, \alpha-\mathrm{Fe}_{6} \mathrm{Ga}_{5}$ и $\beta-\mathrm{Fe}_{6} \mathrm{Ga}_{5}$. Исследование кинетики протекания фазовых переходов для сплавов $\mathrm{Fe}-\mathrm{Ga} \mathrm{c}$ содержанием галлия 17.5-19.5 at.\% представлено в работе [8]. Зарождение фазы $\mathrm{L1}_{2}$ происходит при длительной изотермической выдержки в интервале температур $450-550^{\circ} \mathrm{C}$ и максимальное содержание фазы $\mathrm{L}_{2}$ co- ставляет около $25 \%$ для сплава $\mathrm{Fe}-19.5 \mathrm{Ga}$. Схожие исследования эволюции объемной доли фазы $\mathrm{L1}_{2}$ для сплава $\mathrm{Fe}-27.4 \mathrm{Ga}$ были выполнены в работе [9]. Показано, что фаза $\mathrm{L}_{2}$ формируется в виде кластеров и ее содержание достигает $100 \%$ в интервале температур $400-500^{\circ} \mathrm{C}$ в течении длительного отжига порядка $300 \mathrm{~h}$.

Сплавы на основе $\mathrm{Fe}-\mathrm{Ga}$ исследованы в ряде теоретических работ [10-14]. В работах [10-12] с помощью программных пакетов VASP и SPR-KKR исследованы структурные и магнитные свойства сплавов $\mathrm{Fe}_{100-x} \mathrm{Ga}_{x}$. В работе [10] представлена теоретически полученная фазовая диаграмма для сплава $\mathrm{Fe}_{100-x} \mathrm{Ga}_{x}$ $(x=0-31.25 \%)$. Авторами работы указано, что в исследуемом сплаве наблюдаются следующие фазовые переходы: $\mathrm{D0}_{3} \rightarrow \mathrm{A} 2$ в области концентраций галлия от 6.25 до 18.75 at. $\%$; $\mathrm{DO}_{3} \rightarrow \mathrm{L}_{2} \rightarrow \mathrm{A} 2(18.75<x \leq 21.88$ at.\%); $\mathrm{L} 1_{2} \rightarrow \mathrm{D} 0_{19} \rightarrow \mathrm{B} 2 \rightarrow \mathrm{A} 2(21.88<x \leq 31.25$ at.\% $)$ и для области концентраций галлия до значений 6.25 наблюдается только фаза А2. Работы $[13,14]$ посвящены теоретическому моделированию структурных превращений в сплавах Fe-Ga. Показано, что в данных сплавах наблюдается трансформация путем бездиффузоионной реконструкции ОЦК в ГЦК структуру высокотемпературной равновесной фазы в равновесную $\mathrm{L1}_{2}$ фазу. В работе [14] отмечено, что сплавы с концентрацией галлия 15-20 at.\%, обладают высокой магнитострикцией и это обусловлено индуцированным магнитным полем поворотом осей наночастиц.

Необходимо отметить, что большой интерес представляет исследование фазовых переходов порядок-беспорядок. Например, в работе [15] исследован переход 
порядок-беспорядок для сплавов $\mathrm{Cu}-\mathrm{Zn}-\mathrm{Al}$ с ОЦК решеткой, в которых наблюдается эффект памяти формы. Экспериментальные результаты сопоставлены с данными, полученными путем моделирования методом МонтеКарло. Авторы работы получили хорошее согласие экспериментальной температуры перехода и температуры перехода, полученной в результате моделирования методом Монте-Карло. Это свидетельствует о том, что метод Монте-Карло может быть использован для моделирования фазовых превращений в других сплавах, в частности в сплавах $\mathrm{Fe}-\mathrm{Ga}$.

Настоящая теоретическая работа посвящена исследованию кинетики фазовых переходов порядок-беспорядок в сплаве $\mathrm{Fe}_{80.5} \mathrm{Ga}_{19.5}$ с помощью метода Монте-Карло.

\section{2. Детали расчетов}

Моделирование фазовых переходов в сплаве $\mathrm{Fe}_{80.5} \mathrm{Ga}_{19.5}$ выполнено на кубической решетке типа $\mathrm{DO}_{3}$, состоящей из четырех взаимопроникающих ГЦК-подрешеток (I-IV) (см. рис. 1).

Для определения той или иной фазы использовался вероятностный подход к оценке расположения атомов $\mathrm{Ga}$ или $\mathrm{Fe}$ в тех или иных узлах подрешетки, выраженный через вероятность заполнения $p_{A}^{k}$. Здесь, тип атома определяется индексом $A$, узел подрешетки определяется индексом $k$. Определим ряд условий, характеризующих фазы A2, B2, $\mathrm{L1}_{2}, \mathrm{D0}_{3}$. Для фазы A2 характерно неупорядоченное размещение атомов $\mathrm{Ga}$ и $\mathrm{Fe}$ по узлам подрешеток

$$
p_{A}^{\mathrm{I}}=p_{A}^{\mathrm{II}}=p_{A}^{\mathrm{III}}=p_{A}^{\mathrm{IV}} .
$$

Для фазы В2 соотношение вероятностей имеет вид

$$
p_{A}^{\mathrm{I}}=p_{A}^{\mathrm{II}} \neq p_{A}^{\mathrm{III}}=p_{A}^{\mathrm{IV}} .
$$

Для фазы $\mathrm{D0}_{3}$ справедливо соотношение

$$
p_{A}^{\mathrm{I}}=p_{A}^{\mathrm{II}}=p_{A}^{\mathrm{III}} \neq p_{A}^{\mathrm{IV}} .
$$

Параметры порядка были определены в соответствии со следующими выражениями [15]:

$$
\begin{gathered}
x_{A}=\left(p_{A}^{\mathrm{I}}+p_{A}^{\mathrm{II}}-p_{A}^{\mathrm{III}}-p_{A}^{\mathrm{IV}}\right) / 4, \\
y_{A}=\left(p_{A}^{\mathrm{I}}+p_{A}^{\mathrm{II}}\right) / 2, \\
z_{A}=\left(p_{A}^{\mathrm{III}}-p_{A}^{\mathrm{IV}}\right) / 2 .
\end{gathered}
$$

Проанализировав уравнения (1)-(3), можно получить соотношения параметров порядка для каждой из фаз: для фазы А2 $-x_{A}=y_{A}=z_{A}=0$; для фазы В2 и для фазы $\mathrm{L}_{2}-x_{A} \neq 0$ и $y_{A}=z_{A}=0$; для фазы $\mathrm{D0}_{3}-$ $x_{A} \neq 0, y_{A}=0, z_{A} \neq 0$. Можно видеть, что параметры порядка для структур В2 и $\mathrm{L}_{2}$ аналогичны, поскольку они относятся к одной пространственной группе Pm-3m. Следовательно, для различия фаз $\mathrm{B} 2$ и $\mathrm{L}_{2}$ необходимо воспользоваться дополнительным анализом, поскольку в модельной решетке может также наблюдаться совокупность рассмотренных выше фаз.

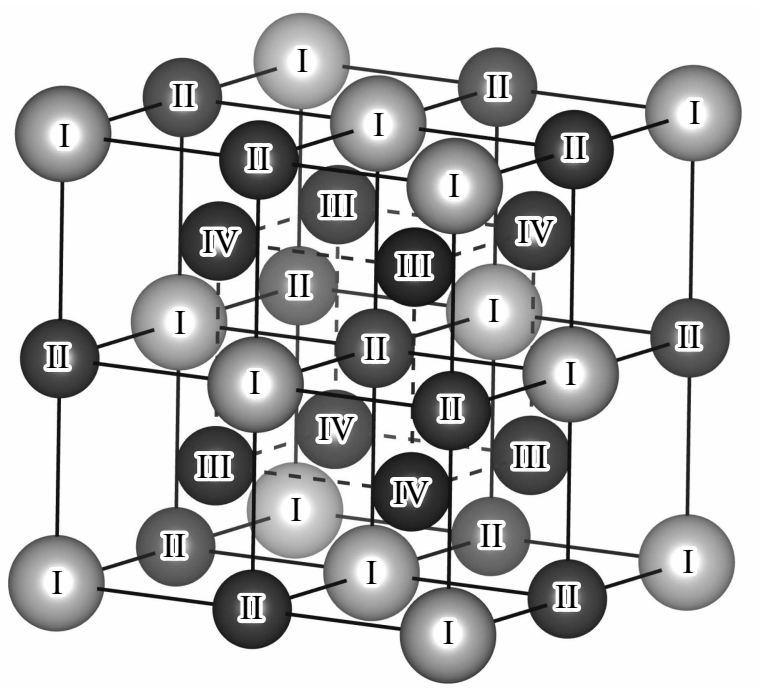

Рис. 1. Исследуемая кристаллическая структура, состоящая из четырех подрешеток (I-IV). Фаза характеризуется вероятностью размещения атомов в определенном узле подрешетки.

В настоящей работе оценка процента содержания фазы $\mathrm{L}_{2}$ проводилась при различных температурах. Для этого в исследуемой структуре, состоящей из 3925 атомов, проводился поиск областей по типу упорядочения $\mathrm{L} 1_{2}$ с соответствующей оценкой процента ее содержания. На рис. 2 изображены характерные положения атомов $\mathrm{Fe}$ и $\mathrm{Ga}$ для фаз $\mathrm{D0}_{3}$ и $\mathrm{L1}_{2}$, на рис. 2, $b$ визуально отображена структура $\mathrm{L1}_{2}$ в виде двух решеток в рамках $\mathrm{D0}_{3}$ структуры. Отметим, что в экспериментальных исследованиях кристаллические структуры часто содержат ряд точечных дефектов, в соответствии с этими данными в нашей работе были рассмотрены два типа точечных дефектов ((1): один тип атома замещается другим; (2): два разноименных атома находятся в позициях друг друга).

Температуры перехода порядок-беспорядок и фазовая стабильность сплавов $\mathrm{Fe}_{80.5} \mathrm{Ga}_{19.5}$ была оценена с помощью метода Монте-Карло. В расчетах использован гамильтониан Блюме-Эмери-Гриффитса (БЭГ) и алгоритм Метрополиса. Моделирование выполнено на кристаллической решетке, состоящей из 3925 атомов с периодическими граничными условиями. Взаимодействие учтено в рамках двух координационных сфер. Рассмотрен процесс охлаждения, т. е. начальной конфигурацией была неупорядоченная фаза, которая наблюдается экспериментально в данном сплаве при высоких температурах. На первом этапе производилось формирование начальной конфигурации расположения атомов в решетке, затем выбирались произвольные узлы в решетке и вычислялись энергии для начальной конфигурации и новой конфигурации, учитывающей перестановку атомов местами. В случае энергетической выгодности новой конфигурации производилось сохранение выгодной конфигурации. Данная процедура выполнялась до тех 

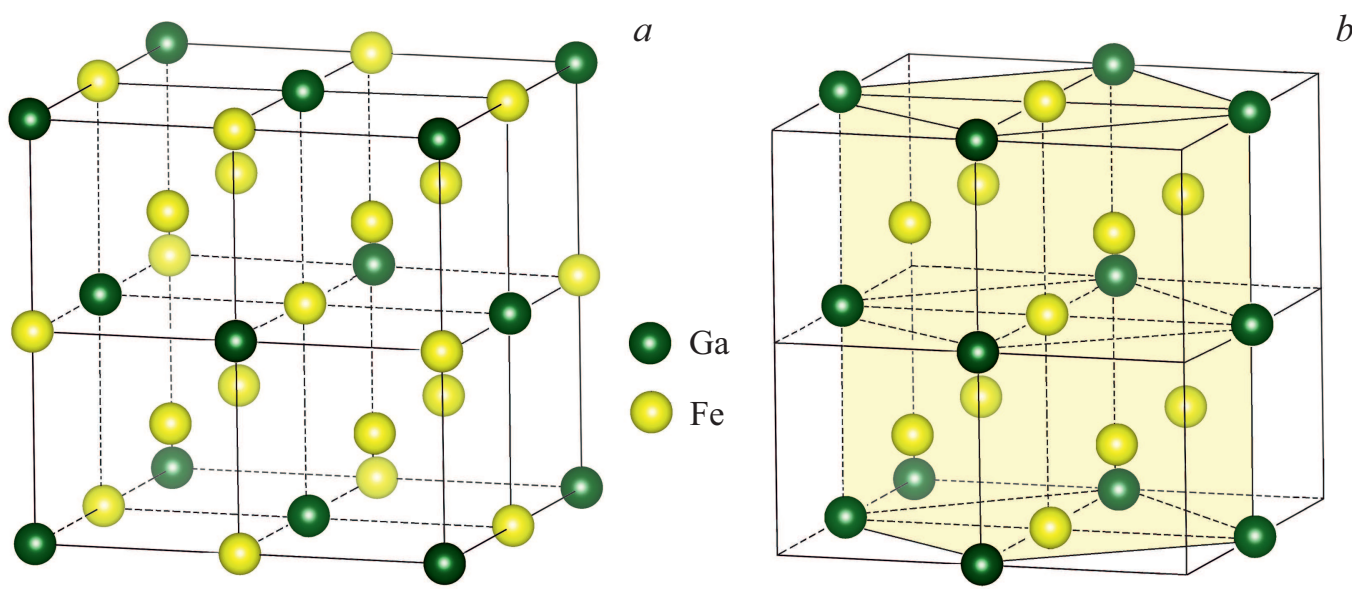

$b$

Рис. 2. Расположение атомов $\mathrm{Fe}$ и $\mathrm{Ga}$ в модельной решетке, соответствующее фазам $\mathrm{D}_{3}(a)$ и $\mathrm{L}_{2}(b)$.

пор, пока не сгенерируется число конфигураций равное числу всех узлов решетки. Данный процесс составляет один шаг Монте-Карло. Кроме того, моделирование проводилось в зависимости от числа шагов МонтеКарло, что позволяет исследовать протекание переходов порядок-беспорядок в зависимости от времени. В качестве единицы времени принимался шаг Монте-Карло.

Гамильтониан БЭГ имеет вид [15]:

$H=\sum_{\langle i j\rangle}^{n n}\left\{J_{1} \sigma_{i} \sigma_{j}+K_{1} \sigma_{I}^{2} \sigma_{j}^{2}\right\}+\sum_{\langle i j\rangle}^{n n n}\left\{J_{2} \sigma_{i} \sigma_{j}+K_{2} \sigma_{I}^{2} \sigma_{j}^{2}\right\}$,

где значение $\sigma$ спиновой переменной связно с расположением атомов галлия и железа в подрешетках, значение $\sigma=-1$ ассоциировано с атомом галлия, $\sigma=1 \mathrm{c}$ атомом железа. Представленные в выражении величины $J_{1}, J_{2}$, $K_{1}, K_{2}$ вычислялись в соответствии со следующими формулами:

$$
\begin{gathered}
K_{k}=-\frac{W^{(k)}}{4}, \\
J_{k}=\frac{W^{(k)}}{4} .
\end{gathered}
$$

Здесь значение $k$-номер координационной сферы (принимает значения $k=1,2), W-$ энергия связи.

Для теоретического моделирования кинетики протекания процессов в сплавах $\mathrm{Fe}-\mathrm{Ga}$ мы рассматриваем энергию связи, зависящую от шагов Монте-Карло, что является аналогом времени отжига в экспериментальной работе [8]. Зависимости значений энергий связи от числа шагов Монте-Карло представлены на рис. 3 . Значения энергий связи в работе были оценены в соответствии с энергиями формирования, полученными из $a b$ initio расчетов свойств основного состояния данной композиции [10-12]. Значение энергии формирования соответствует состоянию системы при максимальном значении термолизации. Так как система со временем приходит в свое равновесное состояние, то значения энергий связи должны быть зависимыми от скорости охлаждения. Значения энергий связи получены путем воспроизведения температур перехода, соответствующих экспериментальным. Возможность фазового перехода оценивалась путем анализа изменения параметров порядка согласно уравнениям (1)-(4).

В настоящей теоретической работе были проведены два этапа моделирования фазовых превращений в соответствии с экспериментальной работой [8]. Первый этап связан с моделированием температурных зависимостей параметров порядка, характеризующих фазовые переходы между фазами $\mathrm{A} 2, \mathrm{~B} 2, \mathrm{D0}_{3}$ и $\mathrm{L}_{2}$ при разной скорости охлаждения (т. е. при разном количестве шагов Монте-Карло). Второй этап моделирования аналогичен процессу длительного отжига. Сначала выполнялось охлаждение модельной решетки с $1500 \mathrm{~K}\left(1227^{\circ} \mathrm{C}\right)$ до $800 \mathrm{~K}\left(527^{\circ} \mathrm{C}\right), 750 \mathrm{~K}\left(477^{\circ} \mathrm{C}\right)$ и $450 \mathrm{~K}\left(177^{\circ} \mathrm{C}\right)$ при постоянном значении энергии связи $W$, затем выполнялось

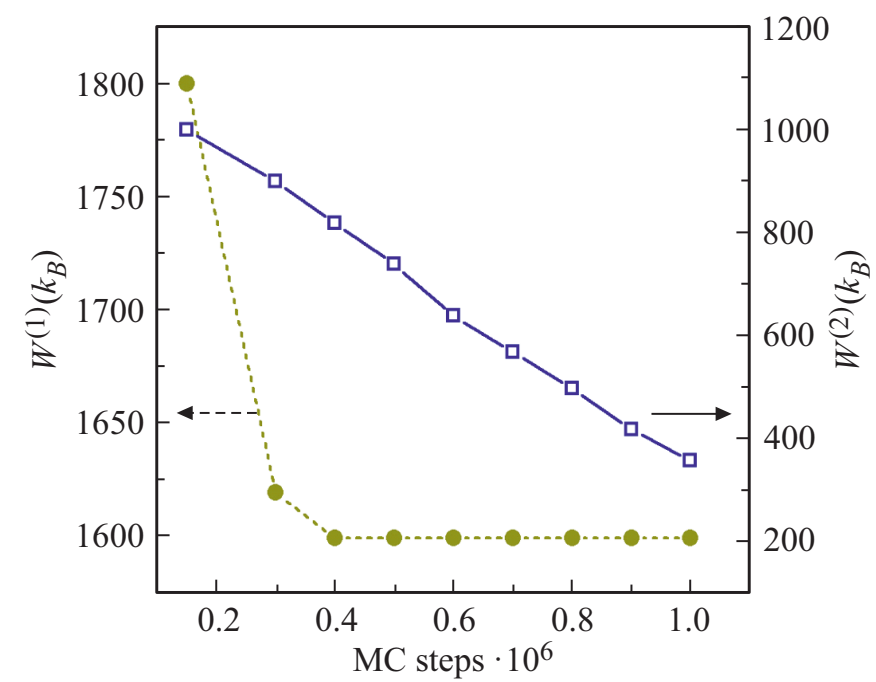

Рис. 3. Зависимости значений энергий связи $W$ от числа шагов Монте-Карло (MC steps). 

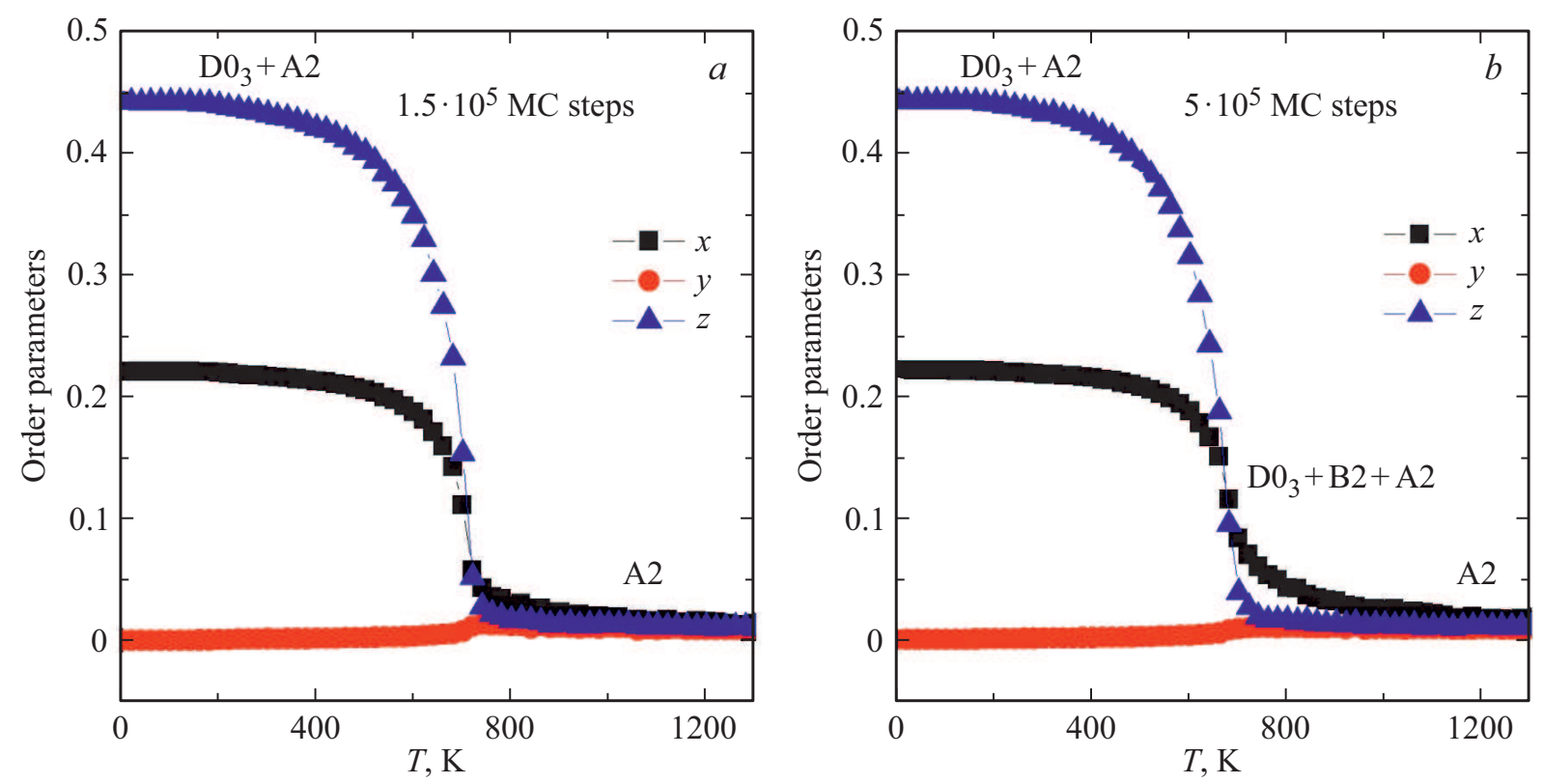

Рис. 4. Кривые изменения параметров порядка при изменении температуры для $a-1.5 \cdot 10^{5}$ шагов Монте-Карло; $b-$ $5 \cdot 10^{5}$ шагов Монте-Карло.

последующее изотермическое моделирование системы при изменении энергии связи в зависимости от числа шагов Монте-Карло.

\section{3. Результаты вычислений и обсуждение}

Рассмотрим результаты моделирования температурных зависимостей параметров порядка, характеризующих фазовые переходы между фазами $\mathrm{A} 2, \mathrm{~B} 2, \mathrm{D0}_{3}$ и $\mathrm{L}_{2}$ при разной скорости охлаждения (см. рис. 4.). Из полученных данных можно сделать вывод о том, что при высоких температурах наблюдается неупорядоченная фаза А2, что согласуется с имеющимися в литературе экспериментальными данными [6]. Из анализа соотношения параметров порядка для количества шагов Монте-Карло равного $1.5 \cdot 10^{5}$ (высокая скорость охлаждения) следует фазовый переход из фазы А2 в некую смешанную фазу, содержащую долю А2 фазы. Рассмотрение низкотемпературной области позволяет сделать заключение, что в данном случае имеет место смешанная фаза с преобладанием фазы $\mathrm{D0}_{3}$, поскольку значение параметра порядка $z<0.5$, тогда как чистая фаза $\mathrm{D}_{3}$ характеризуется параметром порядка $z=0.5$. $\mathrm{B}$ идеале, чистая фаза $\mathrm{D0}_{3}$ должна наблюдаться только в стехиометрическом составе $\mathrm{Fe}_{75} \mathrm{Ga}_{25}$.

Наличие смешанных фаз в представленных расчетах говорит о необходимости дополнительных исследований, направленных на уточнение фазового состава (процентного содержания фазы $\mathrm{L1}_{2}$ ) при изменении температуры и скорости охлаждения сплава. Согласно экспериментальной работе [8] присутствие $\mathrm{L1}_{2}$ фазы в сплаве $\mathrm{Fe}_{80.5} \mathrm{Ga}_{19.5}$ зависит от скорости охлаждения материала и температуры, и ее температурная область лежит в диапазоне от 400 до $550^{\circ} \mathrm{C}(673-823 \mathrm{~K})$. Таким образом, в соответствии с экспериментом [8] второй этап моделирования был аналогичен процессу длительного изотермического отжига. В данном случае модельная система охлаждалась до определенной температуры при постоянном значении энергии связи, и выполнялось последующее изотермическое моделирование системы при изменении энергии связи в зависимости от числа шагов Монте-Карло. При заданном количестве шагов Монте-Карло (от $1 \cdot 10^{5}$ до $1 \cdot 10^{6}$ шагов) проводилась оценка содержания объемной доли фазы $\mathrm{L}_{2}$ в модельной решетке. Данный анализ позволил исследовать процесс эволюции областей фазы $\mathrm{L1}_{2}$ в модельной ячейке при заданной температуре.

На рис. 5 представлены схемы распределения фазы $\mathrm{L}_{2}$ и смешанной фазы $\mathrm{D0}_{3}+\mathrm{A} 2+\mathrm{B} 2$ в объеме исследуемой структуры при температуре $T=750 \mathrm{~K}$ и заданном количестве шагов Монте-Карло $\left(4 \cdot 10^{5}, 6 \cdot 10^{5}\right.$, $\left.10 \cdot 10^{5}\right)$. Как можно видеть из представленных данных, изначально фаза $\mathrm{L}_{2}$ формируется преимущественно небольшими локализованными кластерами, затем наблюдается увеличение как их объемов, так и количества с ростом числа шагов Монте-Карло. Данное наблюдение согласуется с имеющимися экспериментальными данными [8]. Отметим, что максимальные значения содержания фазы $\mathrm{L}_{2}$ составляют $10 \%$. При этом, фаза $\mathrm{L} 1_{2}$ преимущественно формируется кластерами, состоящими из нескольких решеток. Охлаждение сплава до $450 \mathrm{~K}$ и его последующая изотермическая выдержка показывают отсутствие фазы $\mathrm{L1}_{2}$ в модельной решетке, что также соответствует экспериментальным данным [8]. 


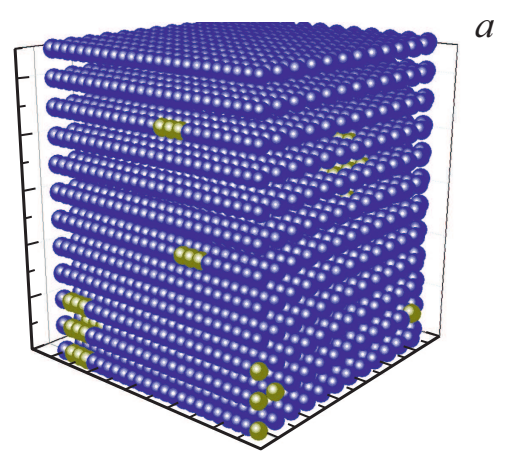

$4 \cdot 10^{5}$ Monte Carlo steps

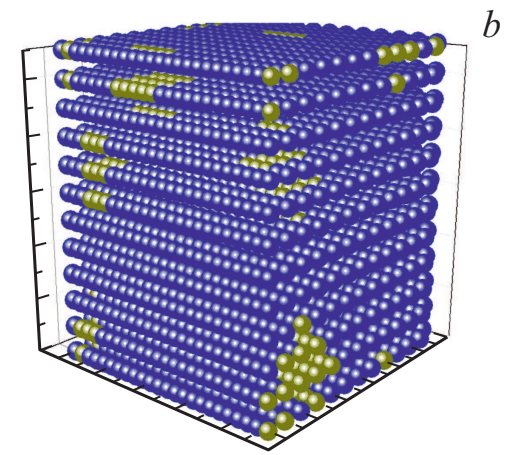

$6 \cdot 10^{5}$ Monte Carlo steps

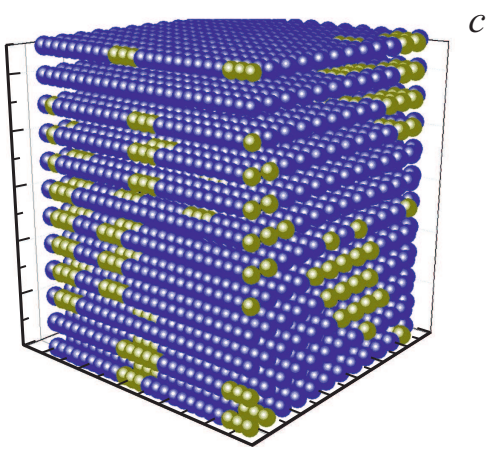

$10 \cdot 10^{5}$ Monte Carlo steps

$$
\text { - Structure area } \mathrm{Ll}_{2} \quad \bullet-\text { Structure area } \mathrm{D}_{3}+\mathrm{A} 2+\mathrm{B} 2
$$

Рис. 5. Результаты моделирования процесса распределения фазы $\mathrm{L}_{2}$ и смешанной фазы $\mathrm{D} 0_{3}+\mathrm{A} 2+\mathrm{B} 2$ в объеме исследуемой структуры при $T=750 \mathrm{~K}$. Количество шагов Монте-Карло составило $\left.4 \cdot 10^{5} a\right), 6 \cdot 10^{5}(b), 10 \cdot 10^{5}(c)$.

На основании расчетов первого этапа моделирования определены температуры фазовых переходов и построена фазовая диаграмма, представленная на рис. 6. Температуры фазовых переходов, полученные при моделировании фазовых превращений в зависимости от скорости охлаждения, обозначены на диаграмме черными квадратами, тогда как доля фазы $\mathrm{L1}_{2}$ при различных температурах представлена квадратными пустыми символами. При построении фазовой диаграммы критерием наличия фазы $\mathrm{L}_{2}$ считается значение больше $1 \%$.

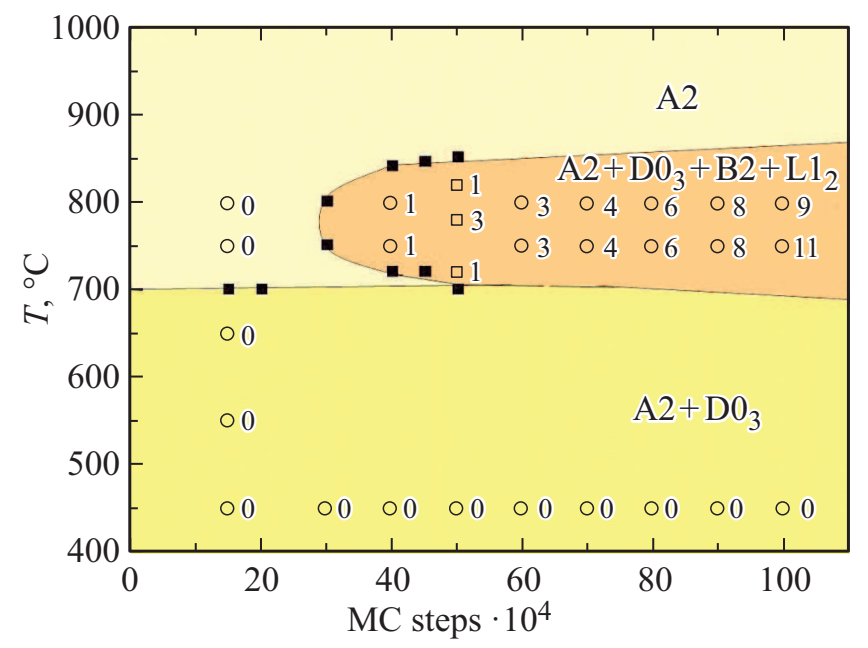

Рис. 6. Теоретически полученная фазовая диаграмма для сплава $\mathrm{Fe}_{80.5} \mathrm{Ga}_{19.5}$. Температуры фазовых переходов, полученные на первом этапе моделирования, обозначенные на диаграмме черными квадратами. Вычисленные значения процентов фазы L12 при различных температурах представлены квадратными пустыми символами. Результаты исследования эволюции содержания фазы $\mathrm{L1}_{2}$ при постоянной температуре, полученные в ходе второго этапа моделирования, представлены на фазовой диаграмме числовыми значениями рядом с пустыми круглыми символами и указывают на процент фазы $\mathrm{L1}_{2}$ в исследуемой трехмерной структуре.
В случае высокой скорости охлаждения реализуется фазовый переход $\mathrm{A} 2 \rightarrow \mathrm{D0}_{3}+\mathrm{A} 2$, уменьшение скорости охлаждения влечет к образованию фазы $\mathrm{A} 2, \mathrm{~B} 2, \mathrm{D0}_{3}$ и $\mathrm{L}_{2}$. Моделирование показывает, что фаза $\mathrm{L1}_{2}$ появляется в интервале температур 680-820 К. Более того, доля данной фазы в указанном температурном интервале возрастает с уменьшением скорости изменения температуры. Таким образом, можно сделать вывод о том, что увеличение скорости охлаждения приводит к быстрому протеканию реакций и ряд фаз в процессе перехода не успевают образоваться.

Перейдем к рассмотрению результатов моделирования эволюции содержания фазы $\mathrm{L}_{2}$ при постоянной температуре в зависимости от количества шагов МонтеКарло. В данном случае доля фазы $\mathrm{L1}_{2}$ отображена числовыми значениями рядом с пустыми круглыми символами (см. рис. 6). Как видно из представленных данных, процент содержания фазы $\mathrm{L}_{2}$ также возрастает с увеличением количества шагов Монте-Карло, что можно условно считать аналогией увеличения времени изотермической выдержки согласно экспериментальной работе [8]. В целом наблюдается хорошее согласие между теоретической и экспериментальной фазовой диаграммой.

\section{4. Заключение}

В настоящей работе рассмотрено моделирование кинетики фазовых переходов порядок-беспорядок с помощью метода Монте-Карло и решеточной модели БлюмеЭмери-Гриффитса. Исследования выполнены на трехмерной кристаллической решетке в два этапа: моделирование температурных зависимостей параметров порядка, характеризующих фазовые переходы при разной скорости охлаждения и моделирование изотермического отжига. По полученным данным построена фазовая диаграмма. Высокая скорость охлаждения приводит к 
фазовому переходу $\mathrm{A} 2 \rightarrow \mathrm{D0}_{3}+\mathrm{A} 2$, тогда как ее последующее уменьшение формирует фазы $\mathrm{A} 2, \mathrm{~B} 2, \mathrm{D}_{3}$ и $\mathrm{L}_{2}$. Объемная доля $\mathrm{L}_{2}$ фазы увеличивается с уменьшением скорости изменения температуры. Представленные исследования позволяют сделать вывод о том, что скорость охлаждения может существенно влиять на протекание фазовых реакций и процесс их образования.

\section{Финансирование работы}

Исследование выполнено при финансовой поддержке Российского научного фонда в рамках проекта № 17-72-20022.

\section{Конфликт интересов}

Авторы заявляют, что у них нет конфликта интересов.

\section{Список литературы}

[1] A.E Clark, M. Wun-Fogle, J.B. Restorff, T.A. Lograsso, J.R. Cullen. IEEE Trans Magn. 37, 2678 (2001).

[2] T.A. Lograsso, A.R. Ross, D.L. Schlagel, A.E. Clark. J. Alloys Compd. 350, 95 (2003).

[3] N. Kawamiya, K. Adashi, Y. Nakamura. J. Phys. Soc. Jpn. 33, 1318 (1972).

[4] A.E. Clark, K.B. Hathaway, M. Wun-Fogle, J.B. Restorff, T.A. Lograsso, V.M. Keppens, G. Petculescu, R.A. Taylor. J. Appl. Phys. 93, 10, 8621 (2003).

[5] Q. Xing, Y. Du, R.J. McQueeney, T.A. Lograsso. Acta Mater. 56, 4536 (2008).

[6] O. Kubaschewski. Iron-binary Phase Diagrams. SpringerVerlag, Berlin, Germany (1982). 185 p.

[7] A.K. Mohamed, V.V. Palacheva, V.V. Cheverikin, E.N. Zanaeva, W.C. Cheng, V. Kulitckii, S. Divinski, G. Wilde, I.S. Golovin. J. Alloys Comp. 846, 156486 (2020).

[8] A.K. Mohamed, V.V. Cheverikin, S.V. Medvedeva, I.A. Bobrikov, A.M. Balagurov, I.S. Golovin. Mater. Lett. 279, 128508 (2020).

[9] I.S. Golovin, A.K. Mohamed, I.A. Bobrikov, A.M. Balagurov. Mater. Lett. 263, 127257 (2020).

[10] M.V. Matyunina, M.A. Zagrebin, V.V. Sokolovskiy, O.O. Pavlukhina, V.D. Buchelnikov, A.M. Balagurov, I.S. Golovin. Phase Transitions 92, 101 (2019).

[11] M.V. Matyunina, M.A. Zagrebin, V.V. Sokolovskiy, V.D. Buchelnikov. J. Magn. Magn. Mater. 470, 118 (2019).

[12] M.V. Matyunina, M.A. Zagrebin, V.V. Sokolovskiy, V.D. Buchelnikov. EPJ Web Conf. 185, 04013 (2018).

[13] A.G. Khachaturyan, D. Viehland. Metallurg. Mater. Transact. A 38, 2308 (2007)

[14] J. Boisse, H. Zapolsky, A.G. Khachaturyan. Acta Mater. 59, 2656 (2011).

[15] F. Lanzini, R. Romero, M. Stipcich, M.L. Castro. Phys. Rev. B 77, 134207 (2008).

[16] G. Kresse, J. Furthmuller. Phys. Rev. B 54, 11169 (1996).
[17] J. Perdew, K. Burke, M. Enzerhof. Phys. Rev. Lett. 77, 3865 (1996).

[18] H. Monkhorst, J. Pack. Phys. Rev. B 13, 5188 (1976).

[19] G. Kresse, D. Joubert. Phys. Rev. B 59, 1758 (1999).

[20] P.R. Alonso, G.H. Rubiolo. Phys. Rev. B 62, 237 (2000).

[21] J. Bai, J.M. Raulot, Y.D. Zhang, C. Esling, X. Zhao, L. Zuo. J. Appl. Phys. 109, 014908 (2011).

Редактор Т.Н. Василевская 